\title{
APPLICATION OF MATERIALS AND PROCESS MODELING TO THE DESIGN, DEVELOPMENT AND SUSTAINMENT OF ADVANCED TURBINE ENGINES
}

\author{
William Gostic \\ Pratt \& Whitney, 400 Main Street, East Hartford, CT 06108, USA \\ Keywords: Integrated Computational Materials Engineering (ICME), Process Modeling, Turbine Engine, Turbine \\ Components, F135 Engine, Geared Turbo Fan ${ }^{\mathrm{TM}}$ Engine
}

\begin{abstract}
Turbine engines provide a challenging environment for materials. The temperature, environment, stress and the time durations over which components are exposed are tremendous. Turbine engines, like other energy and power generation systems, are being ever pushed to increase performance and fuel efficiency. The continued drive is requiring both engine architecture and associated materials changes to meet the need for increase capability. Materials are being asked to provide increased temperature capabilities to support enhanced engine cycles, while at the same time are being required to be lighter weight for increased operational speeds or engine performance. Likewise advances in materials and or materials processing may enable components with reduced engine part count, each designed to more efficiently convert fuel into thrust. These challenges are requiring new materials, and new and novel approaches to apply existing materials.
\end{abstract}

An approach being taken to meet these challenges is through further integration of materials, processes and design functions. Integration of material and manufacturing process design efforts seamlessly with component design processes requires new tools and methods. Integrated computational materials engineering (ICME) is providing the path to holistic linking of material, process and component design optimization. Materials models are providing enhanced materials design definitions by enabling greater physics-based understanding of the fundamental behavior of materials. Compilations of materials and process models effectively "map the genome" of materials and greatly expand the ability for component designer to fully utilize the capabilities of engineered materials, whether emerging new materials or existing materials. This approach to integrated materials, process and design engineering will be the path to enable major changes in future advanced turbine systems. These tools and methods have already made significant impact on current and emerging engine systems.

\section{Introduction}

Turbine engines are continuing to evolve to support increased customer requirements for performance and efficiency. The advancement in engine system capabilities is closely linked to changes in system architecture, component designs and materials requirements. Materials continue to play a major role in the capabilities for turbine engines and often enable significant improvements in operational performance and or durability as evidenced by the F135 engine for the F-35 Joint Strike Fighter or the Geared Turbo Fan $(\mathrm{GTF})^{\mathrm{TM}}$ for the Airbus A320 NEO.

The challenge we have today is to more rapidly enable change in system and component design with new materials or more complete use of existing materials. This challenge can be readily seen through the legacy means of defining a materials capability in the traditional forms of materials specifications, design allowable, and specific print or manufacturing process control requirements. Evolution of how we define materials may be the path toward the ability to further exploit the full capabilities of materials. Materials and process modeling is helping to provide a new means of defining materials and are being applied at an ever increasing rate to design activities and associated materials and process optimization efforts.

Traditional development approaches for newer, more advanced turbine engines often required development of new, more advanced materials. Development of new materials is both costly and time consuming, and has been primarily conducted through empirical trial and error methods and often out of time sync with new systems designs. Substantial physical manufacturing and testing of materials has been required to establish a mechanical property capability definition for a material based on the analysis of a compilation of test data from a range of component configurations and processing conditions. Traditional materials definitions for design system application resulted in materials design curves that represent a statistically derived lower limit on each critical material 
property. The material samples tested and included in the design database were developed based on a material specification, which described to a given tolerance, the chemistry, product form and potentially limited processing window requirements. The data that were generated based upon the materials specification were considered to be of the same pedigree and would all be included in the single population analyzed for the design curve minimum property values. As is known to the materials community, the wide scatter in observed materials properties are a result of specific and often known causes, such as local strain, cooling rate, texture, etc., which are not often taken into account in the analysis of the specific single material population for the material definitions.

As modeling and simulation tools have become available throughout industry and to the design community, further refinement of materials definitions has occurred. Component location-specific properties can now be defined through fine-scale gradients in properties or by more simplistic zoning of volumes within components which are defined by different materials requirements and capabilities. Materials and process modeling tools provide for enhanced understanding of the physics which drive the evolution of materials properties on a location-specific basis and enable this ability to more discreetly define final mechanical properties on a local basis within components.

Materials and process models are becoming closely linked to various stages of the design process. Conceptual design processes provide needed input to the materials community regarding future requirements for materials. These requirements are no longer vague, but rather are in the form of detailed component requirements spatially distributed about notional components. Designers and materials engineers can utilize materials and process models to define a notional requirement for the material that will fulfill the future requirements. This upfront interactive approach effectively enables materials engineers to develop a virtual material with chemistry, microstructure and processing requirements that produce a virtual set of mechanical property capabilities that can then be applied to the design and analysis of conceptual components. If the virtual material capabilities meet the needs for the future requirements, focused material development can then be initiated with a very focused scope and well defined set of materials parameters to achieve the desired capabilities. This was the goal of the DARPA Accelerated Insertion of Materials (AIM) program, and which was successfully demonstrated. [1]

Preliminary design functions occur to establish the bounds on the overall engine architecture and sub-system requirements based on overall system performance targets, including fuel efficiency, thrust, weight and cost. This point in the design process is critical for materials interactions. Enhanced materials definitions, which include the synergy between the inherent material capability and the strong influence of component configuration and manufacturing methods on the component location-specific properties, are needed to enable large potential architecture changes. Changes in the overall engine cycle and or architecture can only effectively be accomplished early in the preliminary design process. If a material can enable architecture change, this often results in huge overall system improvements in efficiency, performance and/or cost.

Figure 1 shows a cross-sectional schematic of the F135 military turbine engine. This unique architecture along with the materials and associated manufacturing processes result in an engine capable of supersonic operation as well as powered lift short take off and vertical landing (STOVL) operation.

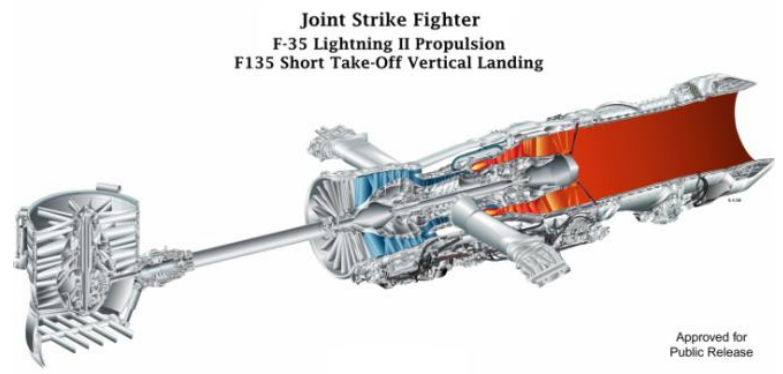

Figure 1. Schematic showing a cross-sectional view of the F135 military turbine engine.

Figure 2 shows a schematic of an advanced commercial geared turbo fan engine, where the fan stage and low pressure turbine module are no longer directly coupled. This enables operating each section of the turbine engine within its optimal efficiency range via light weight, durable engine architectures. The ability to decouple specific engine modules eliminates the constraint one module has on another regarding engine operating conditions thereby enabling significant improvements in fuel efficiency and noise characteristics. With the fan decoupled from the low pressure compressor (LPC) and low pressure turbine (LPT), the LPC and LPT can operate at higher speeds, resulting in improved efficiency. Whereas the fan can be operated at lower speeds utilizing non-conventional materials and fabrications methods. The result is a lighter weight fan with improved propulsive efficiency generating a significantly lower noise signature. This new operating capability now allows materials to be utilized to their fullest for each specific module. 


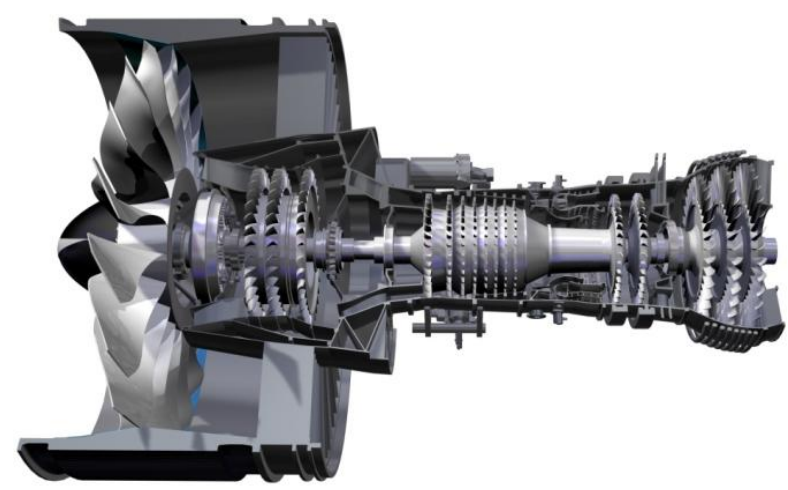

Figure 2. A cross-sectional view of the Geared Turbo Fan ${ }^{\mathrm{TM}}$ engine.

Detailed design processes are also important for materials and process selection and deployment. As architectures change, the influence of subtle materials changes can be enormous. New engine architectures are now more effective in taking advantage of new lighter weight and higher temperature materials. Examples of various aspects of conceptual, preliminary and detailed design efforts related to materials and process development and selection will be further covered throughout the remainder of this paper along with how materials and process modeling has been influencing and enabling many of these efforts.

\section{Application of Materials and Manufacturing Process Modeling and Simulation Tools}

Materials and process modeling tools have been developed and deployed for specific applications for several decades. The forging industry for example uses deformation simulation to ensure lowest possible materials utilization, but at the same time eliminating risks of forging related material defect formation. Modeling of forging processes was used extensively in the development of the GTF and F135 fan, compressor and turbine so as to optimize material properties as well as to improve the buy to fly ratio of the forgings.

Figure 3 is an example of a typical forging process simulation of a fan blade utilized to optimize production manufacturing processes to ensure final component requirements are achieved. Based on the simulation efforts, optimized input material and intermediate manufacturing configurations were established. Criteria for forging strain locally and globally have been developed to guide the assessment and optimization process.

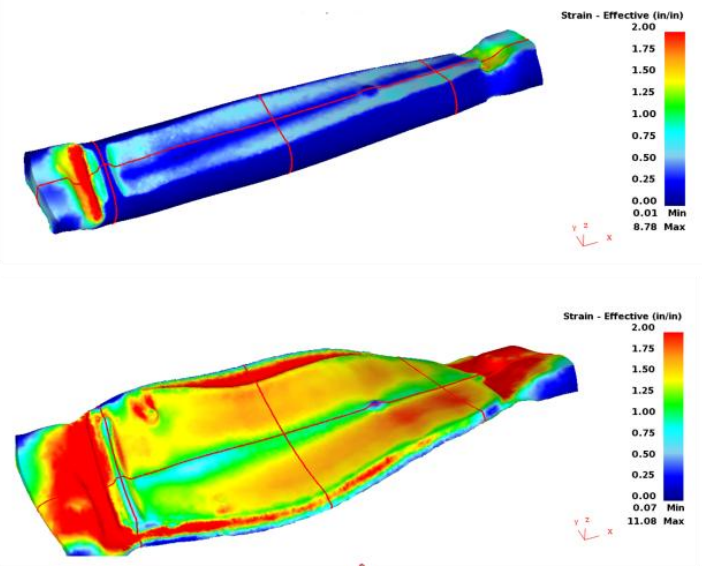

Figure 3. Outputs of process models that detail predicted effective strain following two separate forgings steps for a fan blade. Deformation process simulation is used to ensure optimized strain level, strain rate, and temperatures are achieved during each step of the forging process. (Source: D. Selfridge, Alcoa Forgings, Cleveland, $\mathrm{OH}$ )

The casting industry has employed solidification models to predict and optimize the processing parameters for advanced turbine engine airfoils. In addition to prediction and subsequent elimination of casting defects, such as porosity, hot tear, freckles and recrystallized grain structure, the control of single crystal orientation is critical for optimum material and component capabilities. A range of physicsbased casting and microstructure evolution models have been developed and deployed to production design optimization. Prediction and control of casting microstructure and elimination of casting defects supports enhanced capabilities for sustained service applications as well as for cyclic missions where thermo-mechanical fatigue (TMF) can significantly affect overall component life. Figure 4 shows an example of a typical casting simulation output. This simulation enabled the casting supplier to modify the gating scheme for a mold cluster of commercial engine low pressure turbine blades to avoid a potential hot spot and associated shrinkage porosity. 


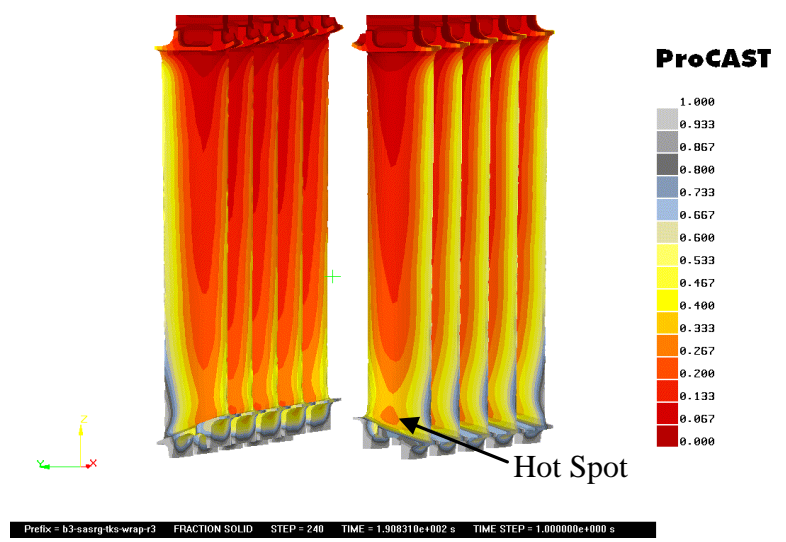

Figure 4. A ProCast ${ }^{\mathrm{TM}}$ simulation for several commercial engine low pressure turbine blades (LPT) showing a potential area of risk for shrinkage porosity at the transition between the blade shroud and airfoil. ProCAST is a trade mark name of the ESI Group.

Modeling of the turbine airfoil castings was used in the development of the F135 engine. Modeling in conjunction with next generation turbine cooling schemes resulted in the F135 engine incorporating the highest turbine inlet temperatures ever achieved in an operational gas turbine engine. Furthermore, casting modeling was used in the development of the specially oriented single crystal turbine blades that enabled improved structural capability and performance of the F135 Low Pressure Turbine. Control of the primary and secondary orientations and tolerances was enabled through model-assisted process design.

Materials and process models are also being applied to other materials, manufacturing and service processes, such as coating application, oxidation and corrosion behavior. Prediction of material pedigree throughout the manufacturing process is important, but the continued evolution of microstructure and mechanical properties during service provide valuable information for life prediction, repair and overhaul requirements. The PROGNOSIS program demonstrated a number of applications where component microstructure (constituent phases and metallurgical damage) can be predicted and applied to component life requirements based on operation and accumulation of mission unique cycles. [2]

It is clear that predictive tools are becoming a necessity for defining and optimizing materials and processes for future engine components. ICME, or the complete holistic application of materials and process models, is providing a tangible path to utilizing materials as an additional degree of freedom as compared to historical approaches where materials were considered a constant single minimum design allowable value throughout the entire volume of a component.

\section{Application of ICME in Support of Advanced Turbine Engine Designs}

Pratt \& Whitney has used materials and process modeling extensively in the development of nearly all new and soon to be fielded turbine engines. Several examples of materials and manufacturing process modeling and simulation applications include:

1. Fan blade materials and manufacturing processes

2. Disk, shaft and case alloy, forging, heat treatment, joining and machining processes

3. Airfoil alloy and process designs

Fan blade assembly and joining processes. Heavily contoured and often hollow, titanium fan blades provide a unique capability for very high speed fan blade applications. These components are processed by means of complex thermo-mechanical processing to produce the required shape, join components into full assemblies and produce optimum microstructure to produce the desired performance capabilities, including strength, stiffness and fatigue.

Pratt \& Whitney employs materials and process modeling to support structural and thermal analyses of manufacturing equipment, tooling systems and components for a range of critical fan blade manufacturing processes including forging, linear friction welding (both OEM and repair processes), inertia friction welding, diffusion bonding and forming of hollow fan blades.

The forging of titanium alloys can produce strain induced porosity (SIP) if local strains and strain rates are not carefully controlled. SIP is of concern in titanium gas turbine engine components because it can act as the site for fatigue crack initiation and significantly impact part durability. At $\mathrm{P} \& \mathrm{~W}$, studies have been performed to correlate strain and strain rates as a function of temperature on the formation of SIP. These studies were conducted using interrupted tensile tests to document the level SIP formed for a given strain rate and strain. The tensile test was modeled using DEFORM ${ }^{\mathrm{TM}}$ to establish a correlation between porosity area fraction and a model defined material damage parameter. Once established, the predicted damage parameter was applied to the development of deformation processes for titanium gas turbine engine components to provide component location-specific understanding of SIP formation. Figure 5 shows the predicted damage parameter 
for various locations along the length of a Ti6-4 tensile specimen. Also shown is the observed post test porosity levels, which were measured using image analysis techniques.

A.
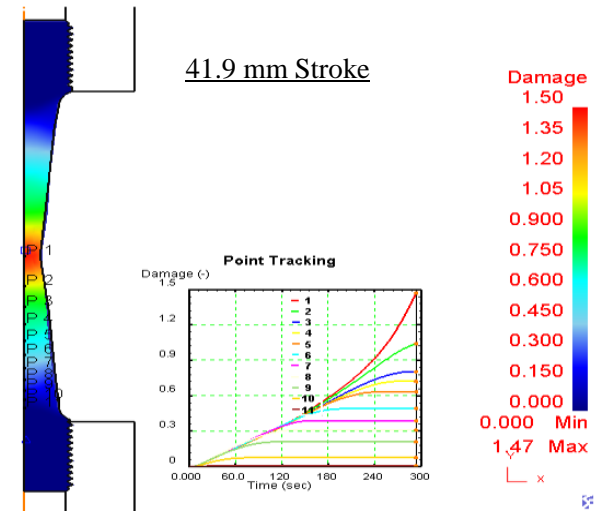

B.

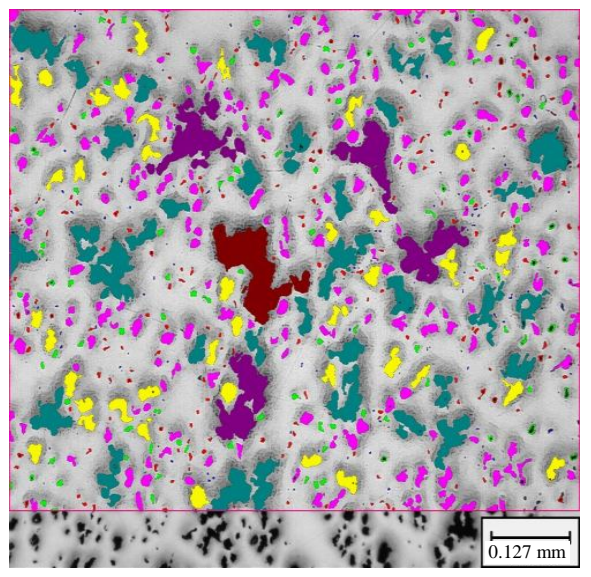

Figure 5. DEFORM ${ }^{\mathrm{TM}}$ prediction of damage parameter during a tensile test of a Ti6-4 specimen (A). Also shown is the observed post-test porosity level (B). Image analysis was used to quantify the level of porosity. DEFORM is a trade mark name of the Scientific Forming Technologies Corporation.

Advanced application of linear friction welding modeling and simulation has resulted in robust OEM manufacturing processes and maturing repair processes for these high cost, critical assemblies. Pratt \& Whitney uses the linear friction welding (LFW) process to manufacture Integrally Bladed Rotors (IBRs) by friction welding blades onto hubs. The blade is oscillated as a normal or forge load is applied to the weld surfaces, resulting in frictional heating of the surfaces and material flows or "upsets". The oscillation ceases once a target upset is achieved and the material is allowed to cool under the forge load. The resulting weld has material properties equal to or better than those of the parent materials. Accurate prediction of thermal conditions, strain, and final upset based on the controlled input material enabled establishment of a robust, repeatable manufacturing process.

LFW repair process development introduced the additional challenge of needing to support finished hub and stub geometries without causing any damage to critical finished surfaces. Significant effort had been invested into developing methods to meet these challenges. LFW process simulation has also been performed to gain insight into material flow and final weld surface geometry and has lead to successful deployment of this technology into the field.

In addition to the assessment and optimization of metal flow, bonding and final geometry; microstructure and mechanical property models have been applied to various fan components in advanced engines. It is well known that the fatigue properties of alpha-beta titanium alloys are closely linked to microstructure, including size, morphology and volume fraction of phases, and crystallographic texture. Tools have been developed and deployed which predict the evolution of titanium microstructure on a location-specific basis during manufacture, leading to a location-specific understanding of component properties during the design phase. Pratt \& Whitney has developed mechanistic, microstructure-sensitive models that link microstructure of alpha-beta titanium alloys to final mechanical properties, such as fatigue. Figure 6 shows an actual versus predicted plot for Ti6-4 high cycle fatigue strength for various stress ratios. This model predicts fatigue strength very accurately based on microstructural constituent phase size, quantity, and texture.

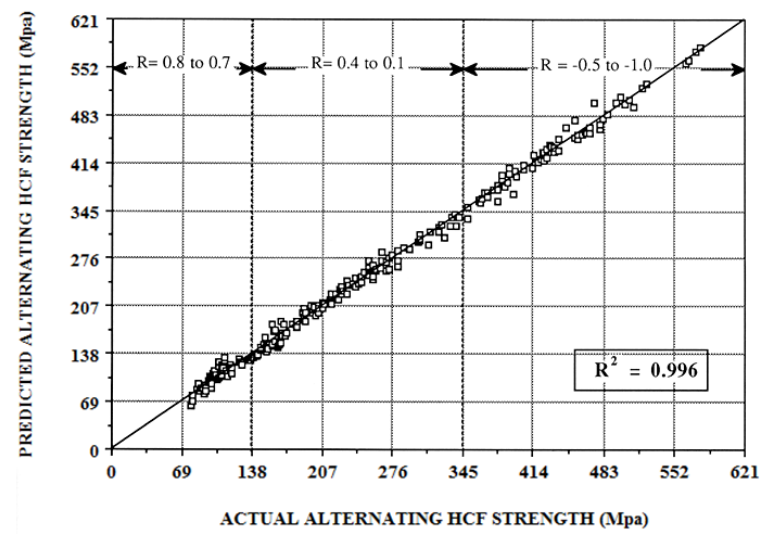

Figure 6. Plot show a comparison of actual versus predicted high cycle fatigue strength for Ti6-4 based on a microstructure-sensitive materials model. 
Disk material and process modeling. At Pratt \& Whitney, material and process modeling of disks includes a number of deformation processes (e.g. forging, extrusion and powder compaction), heat treat processes, joining, machining distortion simulation, microstructure evolution, mechanical property prediction and advanced alloy development.

Disk forging process modeling is applied to a wide range of materials and manufacturing processes, including powder, and conventional cast and wrought alloys by isothermal or conventional press processing. Modeling is used to understand material flow, strain, strain rate and temperature distribution throughout forging processes. Figure 7 shows a typical DEFORM ${ }^{\mathrm{TM}}$ simulation prediction of temperature distribution within a commercial engine IN718 high turbine disk forging during a preliminary preform forging operation. Simulations such as this are used to ensure that the die cavity is completely filled, forging defects do not occur, die loading and stresses do not exceed design limits, and the proper temperature, strain and strain rates are achieved to deliver the desired final microstructure. Materials models are often linked to the continuum process models to provide location-specific microstructure predictions. These in turn can then be used as inputs to subsequent mechanical property models to support design optimization.

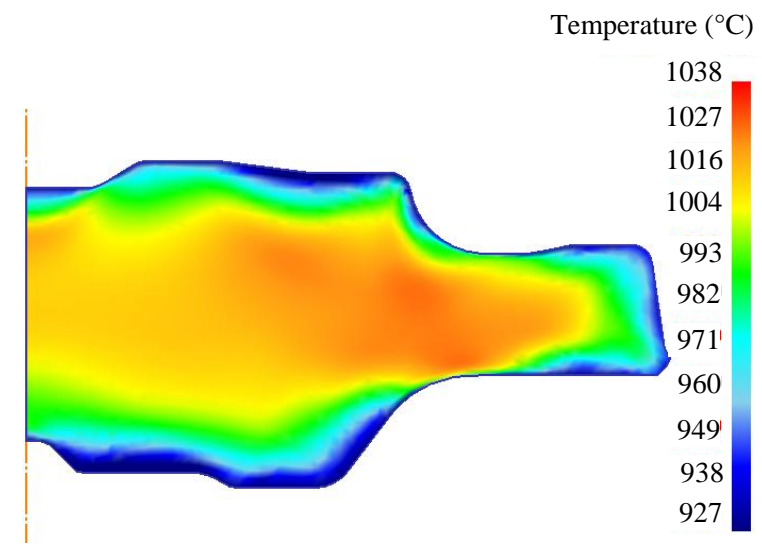

Figure 7. DEFORM ${ }^{\mathrm{TM}}$ simulation prediction of the temperature distribution within a commercial engine IN718 turbine disk forging.

Modeling of the powder consolidation process for powder metallurgy alloys is used to predict relative density level during the compaction process. Extrusion modeling of compacted powder alloys is used to understand strain, temperature and die loading. Tracking the spatial location and orientation of particles (individual point tracking or included secondary objects) is done with varying types of deformation process models.

Disk heat treat process modeling is also applied to isothermal powder and conventional cast and wrought alloy components. Typical applications include alloy and process development, as well as support of design and manufacturing process development and approval. Modeling is used to evaluate heat-up and cooling rates, understand and avoid thermal stress induced cracking, and understand residual stress and distortion during the heat treat process. Figure 8 shows an example of a quench crack prediction for a commercial engine high turbine disk. This figure shows the ratio of predicted thermally driven stress to the material strength value. A pragmatic criterion of one indicates a risk of formation of a quench crack. This type of model can be used to optimize the quench process and part shape to minimize the risk of quench cracking. Figure 9 shows an example of residual stress prediction resulting from the heat treatment of a GTF engine high pressure turbine disk side plate.

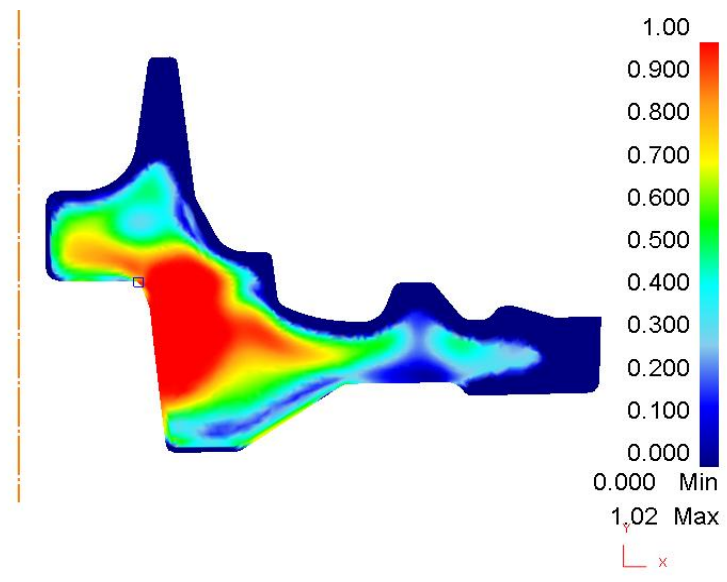

Figure 8. Prediction of quench cracking risk for a commercial engine turbine disk. The plot shows the location-specific prediction of quench cracking parameter.

The prediction of local cooling rate also enables the prediction of microstructure evolution and subsequent prediction of location-specific properties. Figure 10 shows an example of predicted PrecipiCalc secondary gammaprime size distribution throughout the cross-section of a nickel-base superalloy disk. [3]. Figure 11 shows the corresponding contours of predicted yield strength. 


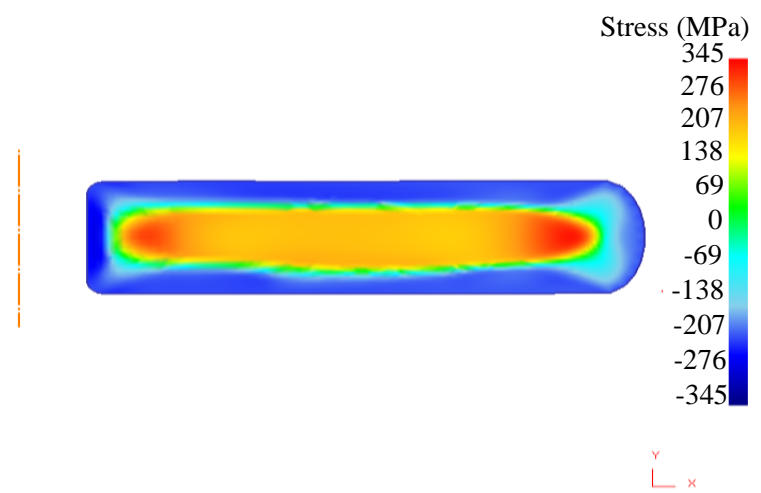

Figure 9. Prediction of residual stress for a GTF engine turbine disk side plate after a series of heat treatment processes. The central region is in tension, whereas the surrounding surfaces are substantially in compression.

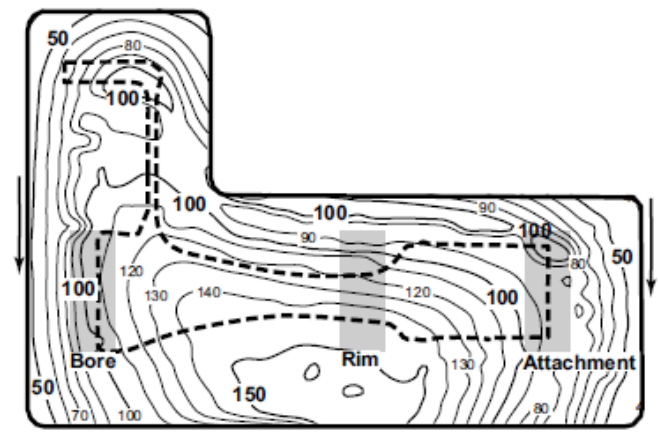

Figure 10. Predicted secondary gamma-prime (in $\mathrm{nm}$ ) precipitated size throughout the cross-section of a nickelbase superalloy disk. [3]

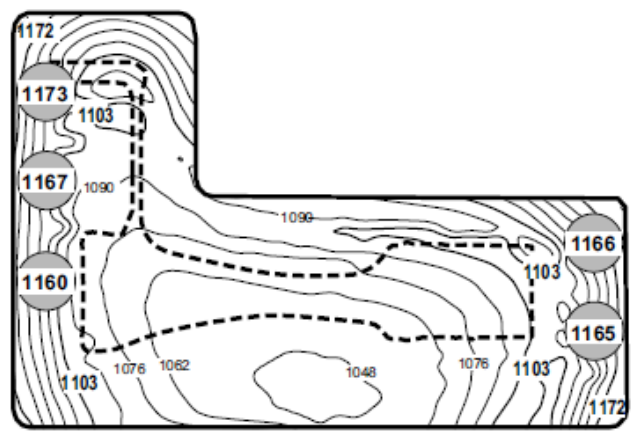

Figure 11. Prediction of yield strength (in $\mathrm{MPa}$ ) at $260^{\circ} \mathrm{C}$ for the same turbine disk shown in Figure 10. [3]

Of critical importance, heat treat modeling provides the ability to predict local cooling paths that set-up bulk residual stresses in final components which impact machining and lifing analysis. Application of models of this type can readily support machining process optimization early in the design phase and can be utilized to analyze impact on fatigue crack growth on a location-specific basis.

Machining distortion modeling is also regularly applied to various gas turbine engine components. It is used to understand how these components distort as material is removed during machining due to the redistribution of residual stress that originated from the heat treat process. This material and process simulation activity is critical for both down-stream manufacturing processes and component field performance. Understanding and controlling these residual stresses can significantly impact manufacturing costs and component performance capabilities. Figure 12 shows the a prediction of distortion that would occur during machining of a superalloy commercial engine high pressure turbine case if steps were not taken to mitigate post heat treat quenching residual stresses.

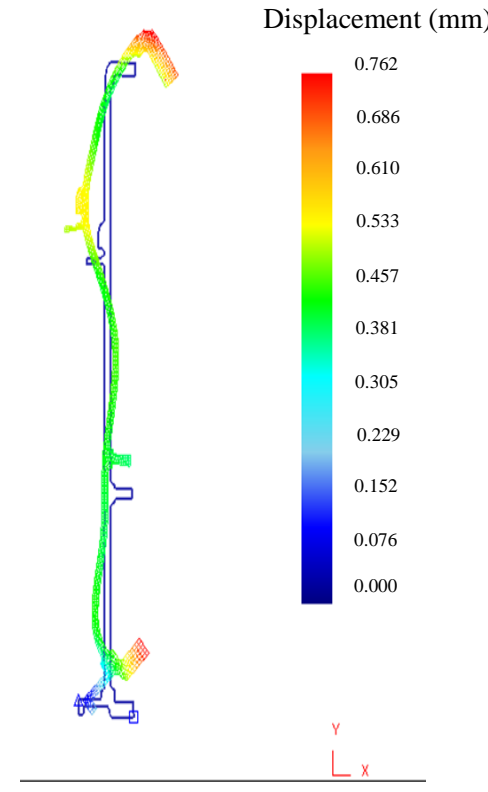

Figure 12. The prediction of machining distortion in a high pressure turbine case resulting from residual stress. The distortion shown in the graphic is numerically exaggerated to highlight the predicted deflection. The magnitude of the deflection is shown in the scale in millimeters.

Understanding and controlling bulk residual stresses is also critical for in-service capabilities. Bulk residual stresses can superimpose their effect onto externally applied stresses. This combined stress state can lead to increased crack growth rates and reduced component lives, making the location-specific prediction and optimization of bulk residual stresses important for disk optimization. 
Process modeling of inertia bonding is also used for joining of adjacent disks to each other. Modeling is used to evaluate the impact of process parameters and tooling on joint heat-up, material flow, upset/flash formation and resulting post bond residual stress. This type of process is used for advanced engine configurations where bolted or otherwise mechanically joined disk stages are joined metallurgically for overall system weight savings. Optimization of bond joints is performed with the aid of modeling and simulation tools. Figure 13 shows an example of an inertia bonding process simulation.

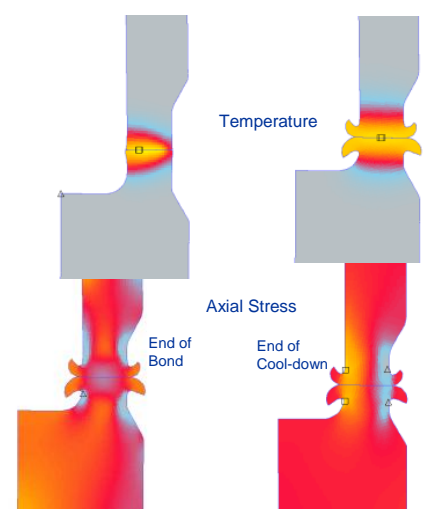

Figure 13 . Inertia welding simulation results showing temperature progression during the process (top images), and residual stresses after completion and cooling of the bonded rotors (bottom images).

Alloy development modeling. Pratt \& Whitney has 40+ years of experience at single crystal nickel base alloy development and a large amassed collection of data. Single crystal superalloy design methods today are largely driven by a combination of empirical and physics-based models. Many composition and process sensitive properties such as density, incipient melting temperature, gamma prime solvus temperature, phase stability, creep resistance and even SRZ sensitivity to coating compositions can be predicted with a high degree of accuracy. In recent years in a response to the escalating price of rhenium, a critical element for high temperature creep strength, two separate alloy development activities were undertaken. Alloy compositions were designed with targets to contain no Re with marginal loss in creep performance and moderate $\operatorname{Re}(3 \%)$ with improved creep performance versus current second generation alloys. Using materials and process models, the alloy development process has been streamlined with development time reduced by $\sim 50 \%$, and with the target compositions selected by screening only a limited number of candidate compositions. This has clearly shown the benefit in development cost, schedule and risk for new alloy development effort.
The capability of modeling is illustrated by Figure 14, showing actual versus predicted single crystal alloy density based on alloy composition. The capability of the established models to predict an intrinsic physical property such as density is very good. Creep property predictions can also be performed by a means of physics-base modeling. Obtaining optimum microstructure formation for maximizing creep resistance of each alloy is a complex function of casting segregation and subsequent heat treatment. Unexplored composition ranges can be readily investigated through application of advanced physics-based modeling methods.

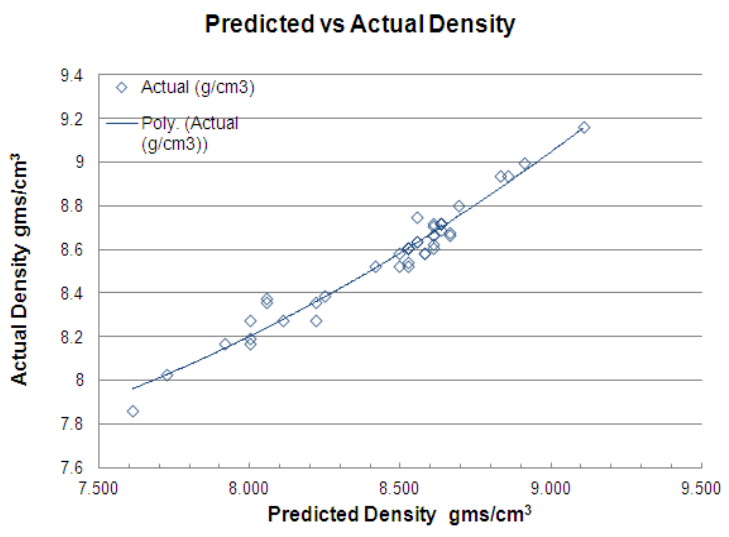

Figure 14. An example of measured versus predicted density for a large number of nickel base superalloys.

Similar to airfoil alloy development, the development of next generation rotor alloys are also being accomplished through the use of modeling and simulation tools. Pratt \& Whitney has developed what is believed to be the highest performance capability disk alloy for applications in next generation military and commercial turbine engines. The time that it has taken Pratt \& Whitney to develop the latest advanced rotor alloy has been greatly reduced, which has also significantly reduced the cost of this overall development process. The development of this alloy with ICME tools has also enabled subsequent linkage of materials models to the component design process to ensure maximum component capabilities.

The critical aspect of utilizing ICME in the development of new engine component designs and the development of new materials is the ability to achieve the component requirement targets and to do so in the same timeframe as new engine design cycles. This has been a challenge, but has clearly been demonstrated in rotor alloy development and new engine deployment efforts at Pratt \& Whitney. 


\section{Unique Examples of ICME for Advanced Engine Requirements}

ICME is being deployed on a more regular, routine basis as part of Pratt \& Whitney engineering standard work. There are many areas were advancements in model development and materials understanding have and are leading to unique applications for specific high performance components and structures, including hybrid disks and advanced composite structures.

Hybrid disks. The realization of hybrid disks with dual microstructures has been enabled by ICME. These advanced rotors have been developed and deployed to advanced engine systems with the support of materials and process modeling. Hybrid disks enable unique property combinations within a single component which enables much higher performance and subsequently the ability for reduced components (stages) to perform the work needed to produce the required thrust. This technology has been applied to the F119 turbine engine and continues to develop as an approach for next generation engines as well.

OMC structural components. The design and optimization of composite materials has always relied on detailed knowledge of fiber/matrix architectures and orientations. Modeling and simulation tools have been developed and are being linked with manufacturing and component design tools which predict the location-specific mechanical properties and component capabilities for organic matrix composites (OMCs). Advanced component applications, such as ducts and frames are being designed and deployed through the use of this linked material, process and design optimization approach.

CMC components. Ceramic matrix composites (CMCs) offer great potential for increasing the performance of specific components and modules within turbine engines. Materials and manufacturing process modeling capabilities are being established to guide the design optimization for various components, such as $\mathrm{CMC}$ combustor liners. Figure 15 shows an example of a CMC combustor liner that has been designed and evaluated in support of material, process and design tool development and validation, as well as the overall assessment of CMC performance capabilities. Like OMCs, material and process models are needed for the prediction and optimization of processing operations, and subsequent material structure and performance capabilities. The ability to predict material anomalies which might limit the performance of a material is critical in order to obtain the maximum use capability and life from manufactured components. Linking process models to microstructure models and then subsequently to mechanical behavior models is clearly a focus that is being aggressively worked to enable rapid insertion of CMC materials.

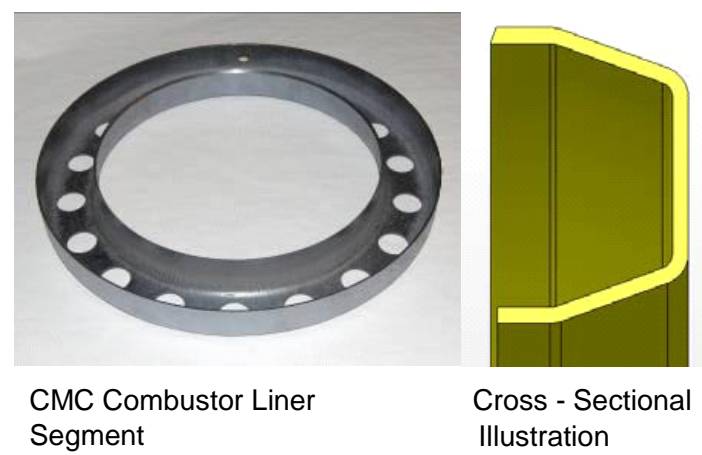

Figure 15. Picture of a CMC combustor liner component that was developed with the support of materials and process modeling tools.

\section{Future Direction of ICME}

As engine requirements continue to evolve, the turbine engine community has also responded. Pratt \& Whitney has been and continues to target improvements in overall systems capabilities through system design and overall architecture advancement. This has been combined with an absolute need for advanced materials capable of performing within the required environments and conditions.

It is clear that overall system improvements will continue to be a combined effort between component/system design and metallurgy/materials. It is also clear that the capabilities of materials or the means by which we define materials must be linked to the manufacturing process and the specific component configuration which synergistically impacts the manufacturing process.

Integrated computational materials engineering (ICME) is an effective approach to an inter-disciplinary linkage of materials, manufacturing and component/systems design. The development and application of ICME will only become stronger as the future progresses. As we see these distinct efforts in linking materials, process and design today, it is envisioned that these activities will become blurred in everyday standard work that is completely interdisciplinary.

ICME tools will become the staple for component and systems design as are current finite element analysis and the much younger computational fluid dynamics methods, which are commonplace in today's design systems. 


\section{Acknowledgements}

DARPA and the U.S. Air Force must be acknowledged for their pioneering efforts and leadership in establishing the modern ICME framework on which much of the aerospace industry is working. The author also acknowledges the contributions of D. Selfridge (Alcoa Inc.), T. Howson (Wyman-Gordon Company), A. Cetel, F. Cohen, Dr. D. Furrer, D. Gynther, A. Haynes, R. Montero, Dr. V. Seetharaman, and $\mathrm{Dr}$. W. $\mathrm{Yu}$, for their contributions toward the preparation of this manuscript.

\section{$\underline{\text { References }}$}

1. D. S. Stolz and J. J. Schirra, "Role of Integrated Computational Materials Engineering in Turbine Rotor Alloy Development", 52th AIAA/ ASME/ ASCE/ AHS/ ASC Structures, Structural Dynamics, and Materials Conference, Denver Colorado, USA, 2011.

2. Materials damage prognosis: proceedings of a symposium held during the Materials Science \& Technology 2004 Conference, J. M. Larsen, Ed., New Orleans, Louisiana, USA, September 26-30, 2004

3. Jou, H. J., Voorhees, P., and Olson, G. B., "Computer Simulations for the Prediction of Microstructure / Property Variation in Aeroturbine Disks", Superalloys 2004, eds. Green, K. A. et al., TMS, Warrendale, PA, 2004, pp. 877 - 886.) 